\title{
ON THE VALUES ASSUMED BY POLYNOMIALS*
}

\author{
HOWARD LEVI
}

The equation

$$
\frac{f(x+h)-f(x)}{h}=k
$$

where $f(x)$ is an integral function and $k$ is an independent complex variable, defines $h$ as a many valued function of $x$ and $k$. The branches of this function $h(x, k)$ possess a simple property when $f(x)$ is a polynomial; and conversely, when the branches of $h(x, k)$ have the property, $f(x)$ must be a polynomial. It is the object of this paper to exhibit the property, prove that it is characteristic for polynomials, and extend the results to polynomials of several variables.

1. Polynomials in one variable. For a polynomial

$$
f(x)=a_{0} x^{n+1}+a_{1} x^{n}+\cdots+a_{n+1}, \quad a_{0} \neq 0, n>1,
$$

equation (1) becomes

$$
h^{n}+\left[(n+1) x+a_{1} / a_{0}\right] h^{n-1}+\cdots-k / a_{0}=0,
$$

and the $n$ branches $h_{i}(x, k)$ of the function $h(x, k)$ satisfy the equation

$$
\sum_{1}^{n} h_{i}(x, k)=-(n+1) x-a_{1} / a_{0} .
$$

For $n=1$ we readily find that $h=-2 x-a_{1} / a_{0}+k / a_{0}$. Hence we have

$$
\frac{\partial}{\partial x} \sum_{1}^{n} h_{i}=-(n+1), \quad n \geqq 1,
$$

as a property of polynomials of degree $n+1$. For $n=0$ no function $h$ is defined by (1).

THEOREM I. Let $f(x)$ be an integral function. If there exist $n$ functions $h_{1}(x, k), \cdots, h_{n}(x, k)$ analytic, distinct, and nonzero on a region in the $x$ plane and a region in the $k$ plane, and which on these regions are such that

and

$$
\frac{f\left(x+h_{i}(x, k)\right)-f(x)}{h_{i}(x, k)}=k, \quad i=1, \cdots, n,
$$

* Presented to the Society, February 26, 1938, under the title $A$ characterization of the values assumed by polynomials. 


$$
\frac{\partial}{\partial x} \sum_{1}^{n} h_{i}(x, k)=-(n+1),
$$

then $f(x)$ is a polynomial of degree $n+1$.

The $n$ functions $h_{i}$ of our hypothesis satisfy

$$
\begin{aligned}
& \left(h-h_{1}\right)\left(h-h_{2}\right) \cdots\left(h-h_{n}\right)=0 \\
& \quad=h^{n}-P_{1} h^{n-1}+\cdots+(-1)^{i} P_{i} h^{n-i}+\cdots+(-1)^{n} P_{n} .
\end{aligned}
$$

In order to prove the theorem we establish relations between the elementary symmetric functions $P_{i}$ and their partial derivatives. Every function $h_{i}$ satisfies the equation

$$
\left(1+\frac{\partial h}{\partial x}\right)=\frac{f^{\prime}(x)-k}{h} \frac{\partial h}{\partial k} .
$$

We may derive this by differentiating both members of (1) with respect to $x$, obtaining

$$
f^{\prime}(x+h)\left(1+\frac{\partial h}{\partial x}\right)=f^{\prime}(x)+k \frac{\partial h}{\partial x}
$$

and with respect to $k$ obtaining

$$
f^{\prime}(x+h) \frac{\partial h}{\partial k}=h+k \frac{\partial h}{\partial k} .
$$

Since $h \neq 0, \partial h / \partial k \neq 0$, and we may solve this last equation for $f^{\prime}(x+h)$; and the substitution of the value thus obtained into the preceding equation yields (4). We shall make use of the fact that $\partial h / \partial k \neq 0$ later in the proof. From (4) we see that

$$
\sum_{1}^{n}\left(\frac{\partial h_{i}}{\partial k} \frac{1}{h_{i}}\right)=\frac{\partial P_{n}}{\partial k} \frac{1}{P_{n}}=\frac{1}{f^{\prime}(x)-k} \sum_{1}^{n}\left(1+\frac{\partial h_{i}}{\partial x}\right)
$$

and since by hypothesis $\sum_{1}^{n} \partial h_{i} / \partial x=-(n+1)$, this reduces to

I

$$
\frac{1}{P_{n}} \frac{\partial P_{n}}{\partial k}=\frac{1}{k-f^{\prime}(x)} \text {. }
$$

We now proceed to deduce a second equation connecting the $P$ 's and their partial derivatives; namely,

$$
\frac{\partial P_{i+1}}{\partial x}=-(n-i+1) P_{i}+\left(k-f^{\prime}(x)\right) \frac{\partial P_{i}}{\partial k},
$$

$$
i=1,2, \cdots, n-1 \text {. }
$$


To accomplish this observe that

$$
\frac{\partial P_{i+1}}{\partial x}+(n-i) P_{i}=\delta h_{1} h_{2} \cdots h_{i} \sum_{j}\left(1+\frac{\partial h_{j}}{\partial x}\right)
$$

where $S$ denotes the sum of the terms arising by choosing the $i$ indices of the $h$-factors in all possible ways among the numbers $1,2, \cdots, n$, and then letting $j$ run through the remaining $n-i$ numbers. The right-hand side of this equation can be written in the form

$$
S h_{1} h_{2} \cdots h_{i}\left\{\sum_{j=1}^{n}\left(1+\frac{\partial h_{j}}{\partial x}\right)-\sum_{j=1}^{i}\left(1+\frac{\partial h_{j}}{\partial x}\right)\right\} \text {. }
$$

With the aid of (2) and (4) the expression now becomes

$$
\begin{aligned}
S h_{1} h_{2} \cdots h_{i}\left\{[n-(n+1)]+\left(k-f^{\prime}(x)\right)\right. & \left.\sum_{j=1}^{i} \frac{1}{h_{j}} \frac{\partial h_{j}}{\partial k}\right\} \\
& =-P_{i}+\left(k-f^{\prime}(x)\right) \frac{\partial P_{i}}{\partial k} .
\end{aligned}
$$

Hence II follows at once.

It will be convenient to change the notation, replacing $i$ by $n-i$. Then

II*

$$
\begin{aligned}
& \frac{\partial P_{n-i+1}}{\partial x}=-(i+1) P_{n-i}+\left(k-f^{\prime}(x)\right) \frac{\partial P_{n-i}}{\partial k} \\
& i=1,2, \cdots, n-1 .
\end{aligned}
$$

From I it follows that

$$
P_{n}=\phi(x)\left(k-f^{\prime}(x)\right)
$$

where $\phi(x)$ is analytic in the given domain of the $x$-plane and does not vanish there. Observe that $P_{n} \neq 0$ since no $h_{i}(x, k)$ vanishes, and, since the left-hand side of $\mathrm{I}$ is analytic in the given domain, $k-f^{\prime}(x)$ cannot vanish there

We proceed to show that $P_{n-i}$ is a polynomial in $k$, at most of degree $i+1$, with coefficients which are analytic functions of $x$ in the given domain, or else that $P_{n-i}$ vanishes identically. We use the method of mathematical induction. First, we know that $P_{n}$ is linear in $k$. Next, observe that from II* it follows that

$$
\frac{\partial}{\partial k} \frac{P_{n-i}}{\left(k-f^{\prime}(x)\right)^{i+1}}=\frac{\partial P_{n-i+1}}{\partial x} \frac{1}{\left(k-f^{\prime}(x)\right)^{i+2}} .
$$

Assume the statement true for $P_{n}, P_{n-1}, \cdots, P_{n-i+1}$. Then the 
right-hand side of this last equation can be represented by partial fractions

$$
\frac{A_{i+2}}{\left(k-f^{\prime}(x)\right)^{i+2}}+\cdots+\frac{A_{2}}{(k-f(x))^{2}},
$$

where the $A_{j}$ are functions of $x$ analytic in the given domain. The fraction whose denominator is $k-f^{\prime}(x)$ is absent, because the degree of the numerator, $\partial P_{n-i+1} / \partial x$ is at most $i$. Hence on integrating we have

$$
\frac{P_{n-i}}{\left(k-f^{\prime}(x)\right)^{i+1}}=-\frac{A_{i+2}}{(i+1)\left(k-f^{\prime}(x)\right)^{i+1}}-\cdots-\frac{A_{2}}{k-f^{\prime}(x)}+\psi(x) .
$$

This completes the proof.

If we substitute $(f(x+h)-f(x)) / h$ for $k$ in (3), and multiply both members by $h$, we have an equation of the form

$$
h^{n+1}+\sum C_{p q r}(x) h^{p} f(x)^{q} f(x+h)^{r}=0, \quad p+q+r \leqq n+1 .
$$

This equation is of positive degree in $f(x+h)$. For, if it were of degree zero, $h$ would depend on $x$ alone and $\partial h / \partial k=0$. We have seen above that $\partial h / \partial k \neq 0$. Thus, for constant $x, f(x+h)$ coincides with a branch of an algebraic function of $x+h$; and since $f$ is integral, the algebraic function is a polynomial, and so is $f$.

There remains only to show that $f$ is a polynomial of degree $n+1$. Since (1) defines $n$ distinct, nonzero elements $h_{i}$ for $f$, the degree of $f$ is not less than $n+1$. In addition, we readily see the degree of $f$ cannot exceed $n+1$. In fact, if we substitute the Taylor expansion of $f(x+h)$ in (5), the resulting polynomial in $h$ must be identically zero, and this can happen only if the degree of $f(x+h)$ in $h$ does not exceed $n+1$.

2. Polynomials in $N$ variables. The above characterization of polynomials of a single variable finds its analogue for polynomials of several variables as follows: The equation (1) is replaced by

$$
\frac{f\left(x_{1}+k_{1} h, x_{2}+k_{2} h, \cdots, x_{N}+k_{N} h\right)-f\left(x_{1}, x_{2}, \cdots, x_{N}\right)}{h}=k
$$

with $x_{1}, \cdots, x_{N}, k_{1}, \cdots, k_{N}, k$ independent complex variables. If we denote by $D$ the operator $k_{1} \partial / \partial x_{1}+k_{2} \partial / \partial x_{2}+\cdots+k_{N} \partial / \partial x_{N}$, we may assert that if $f\left(x_{1}, \cdots, x_{N}\right)$ is a polynomial in $x_{1}, \cdots, x_{N}$, of total degree $n+1$, then there exist $n$ analytic, distinct, nonzero elements

$$
h_{i}\left(x_{1}, \cdots, x_{N} ; k_{1}, \cdots k_{N} ; k\right)
$$

which satisfy $\left(1^{\prime}\right)$ and are such that 


$$
D \sum_{1}^{n} h_{i}=-(n+1) .
$$

This fact is readily established if we expand $f\left(x_{1}+k_{1} h, \cdots, x_{N}+k_{N} h\right)$ by Taylor's theorem, obtaining

$$
\begin{aligned}
& f\left(x_{1}+k_{1} h, \cdots, x_{N}+k_{N} h\right) \\
& \quad=f\left(x_{1}, \cdots, x_{N}\right)+h D f+\cdots+\frac{h^{i}}{i !} D^{i} f+\cdots+\frac{h^{n+1}}{(n+1) !} D^{n+1} f .
\end{aligned}
$$

Thus $\left(1^{\prime}\right)$ may be written

$$
\begin{aligned}
& h^{n}+(n+1) \frac{D^{n} f}{D^{n+1} f} h^{n-1}+\cdots+\frac{(n+1) !}{i !} \frac{D^{i f}}{D^{n+1} f} h^{i-1}+\cdots \\
& \text { and since } D^{n+2} f \equiv 0,
\end{aligned}
$$

$$
D \sum_{1}^{n} h=D\left(-(n+1) \frac{D^{n} f}{D^{n+1} f}\right)=-(n+1)\left(\frac{D^{n+1} f}{D^{n+1} f}\right)=-(n+1) .
$$

THEOREM II. Let $f\left(x_{1}, \cdots, x_{N}\right)$ be an integral function. If there exist $n$ functions $h_{i}\left(x_{1}, \cdots, x_{N} ; k_{1}, \cdots, k_{N} ; k\right)$, analytic, distinct, and nonzero on a region in $\left(x_{1}, \cdots, x_{N} ; k_{1}, \cdots, k_{N} ; k\right)$ space and which on this set are such that they satisfy $\left(1^{\prime}\right)$ and

$$
D \sum_{1}^{n} h_{i}=-(n+1),
$$

then $f$ is a polynomial of total degree $n+1$.

The proof of Theorem II is identical with the proof of Theorem I, where the counterpart of equation (4) is

$$
(1+D h)=\frac{(D f-k)}{h} \frac{\partial h}{\partial k}
$$

and those of I and II are, respectively,

$I^{\prime}$

$$
\frac{1}{P_{n}} \frac{\partial P_{n}}{\partial k}=-\frac{1}{D f-k}
$$

$\mathrm{II}^{\prime}$

$$
D P_{i+1}=-(n-i+1) P_{i}-\frac{\partial P}{\partial k}\left(D_{J}-k\right) .
$$

To establish $\left(4^{\prime}\right)$ from $\left(1^{\prime}\right)$ we operate on both members with $D$, getting 


$$
\begin{aligned}
D f+k D h= & D f\left(x_{1}+k_{1} h, \cdots, x_{N}+k_{N} h\right) \\
= & \sum k_{i} \frac{\partial}{\partial x_{i}} f\left(x_{1}+k_{1} h, \cdots, x_{N}+k_{N} h\right) \\
= & \left(\sum_{i} k_{i} \sum_{j} \frac{\partial}{\partial x_{j}+k_{j} h}\right. \\
& \left.\cdot f\left(x_{1}+k_{1} h, \cdots, x_{N}+k_{N} h\right)\left(k_{j} \frac{\partial h}{\partial x_{i}}\right)\right) \\
& +\sum_{i} k_{i} \frac{\partial}{\partial x_{i}+k_{i} h} f\left(x_{i}+k_{i} h\right) \\
= & \left(\sum_{j} k_{j} \frac{\partial}{\partial x_{j}+k_{j} h}\right. \\
& \left.\cdot f\left(x_{1}+k_{1} h, \cdots, x_{N}+k_{N} h\right)\right)(1+D h) .
\end{aligned}
$$

Differentiating both members of $\left(1^{\prime}\right)$ with respect to $k$, we have

$$
k \frac{\partial}{\partial k} h+h=\left\{\sum k_{i} \frac{\partial}{\partial x_{i}+k_{i} h} f\left(x_{1}+k_{1} h, \cdots, x_{N}+k_{N} h\right)\right\} \frac{\partial h}{\partial k},
$$

and eliminating the term in the bracket yields equation $\left(4^{\prime}\right)$.

Since the operator $D$ behaves for its variables like the operator $d / d x$ for a single variable, all the reasoning of the previous section can, from this point on, be used verbatim, proving Theorem II.

Columbia University 